\title{
Desafíos para la profesionalización del nuevo rol docente universitario
}

\section{Resumen}

El presente artículo tiene por objetivo relacionar las demandas educacionales del nuevo siglo, visando las modificaciones que se hacen necesarias al ejercicio de la docencia universitaria. La principal implicación consiste en la sustitución de presupuestos básicos por procedimientos docentes diferenciados y desenvolvimiento de otros conocimientos, levándose en cuenta los resultados de pesquisas recientes. El texto está dividido en tres abordajes principales. La primera - énfasis para la acción docente presenta resultados de pesquisas recientes sobre la docencia eficaz. La segunda - funciones y tareas de las nuevas atribuciones docentes - se basa en experiencias docentes innovadoras, siendo ilustradas por algunas funciones y tareas-clave que deberían estar presentes en la actuación del profesor. La tercera - el rol docente en nueva visión sistémica - considera docencia como algo desafiante y presenta sugestiones para el avanzo de la profesionalización docente en esa nueva visión.

Palabras clave: Demandas educacionales. Docencia universitaria. Pesquisas. Profesionalización.
Resumo

Desafios para a profissionalização do novo papel docente universitário.

$\bigcirc$ presente artigo tem por objetivo relacionar as demandas educacionais do novo século, com as modificações que se fazem necessárias ao exercício da docência universitária. A principal implicação consiste na substituição de pressupostos básicos por procedimentos docentes diferenciados, além do desenvolvimento de outros conhecimentos, levando-se em conta os resultados de pesquisas recentes.

$\bigcirc$ texto está dividido em três abordagens principais, A primeira - mudanças no desempenho da ação docente apresenta resultados de pesquisas recentes feitas acerca da docência eficaz. A segunda - funções e tarefas das novas atribuições docentes - baseia-se em experiências docentes inovadoras, sendo ilustradas por algumas funções e tarefas-chave que deveriam estar presentes na atuação de professor.A terceira - $\bigcirc$ papel docente em nova visão sistêmica - considera docência como 
algo desafiante e apresenta sugestões para o avanço da profissionalização docente nessa nova visão.

Palavras-chave: Demandas educacionais. Docência universitária. Pesquisas. Profissionalização

\section{Abstract \\ Challenges in the profissionalization of the university teaching's new role}

The objetive of this paper is to relate the educational demands of the new century with the modifications being needed in the university teaching practice. The main implication consists in the substitution of basic presuppositions for differentiate teaching procedures. This in addition to further knowledges development, taking into account the results of recent researchs.

The text is dividid into in three approaches addresses. The firstchanges in the teaching action performance - presents results of recent researches-on the effective teaching. The second - functions and tasks of the teaching-new attributions - is based in inovattive teaching experiences, being illustrated by certain functions and keytasks that should be present in the professor performance. The third - the teaching role in a new systemic vision considers teaching as something challenging and presents suggestions to the advance of teaching profissionalization in that new vision.

Keywords: Educational demands. University teaching. Researches. Profissionalization.
Las demandas educacionales del nuevo siglo comprometen modificaciones sustantivas de la docencia afectando las decisiones principales del rol docente. En lo principal, implica cambiar supuestos básicos de la docencia convencional, hacerse cargo de otras tareas y aplicar varios tipos de conocimientos que se han destacado como importantes en investigaciones recientes sobre la docencia universitaria eficaz.

El nuevo rol docente esta fuertemente afectado por un cambio de énfasis de una docencia centrada en la enseñanza hacia otra en el aprendizaje, con la preocupación por preparar a los estudiantes para una acción social competente y, por la introducción de los ambientes y situaciones de aprendizaje con tecnologías informáticas y de comunicación.

A la luz de algunas experiencias vinculadas con el desarrollo curricular basada en competencias con introducción de tecnologías informáticas y de comunicaciones, en esta presentación, se asume la importancia de profesionalizar la docencia que permita sustentar nuevas funciones y tareas docentes en las universidades.

Además de aplicar su conocimiento experto disciplinario, la profesionalización de la docencia debería posibilitar estar en condiciones de aplicar otros conocimientos que faciliten un proceso formativo que afecten tanto la forma de pensar, como las de sentir y actuar de los estudiantes de acuerdo con las nuevas demandas educacionales.

Esta presentación está organizada en tres secciones principales. En la primera se ilustra el cambio de énfasis para la nueva docencia y algunos resultados de las in- 
vestigaciones acerca de la docencia universitaria eficaz. En la segunda, sobre la base de experiencias innovadoras docentes se ilustran algunas funciones y tareas claves que se deberían desempeñar en el nuevo rol docente y en la tercera, considerando una visión sistémica de la docencia se presentan algunos desafíos y sugerencias para avanzar en la profesionalización en el nuevo rol docente.

\section{Cambios de énfasis para la docencia}

Con la docencia universitaria pretendemos formar a los jóvenes y adultos para una acción social competente en roles sociales de determinados ámbitos profesionales y académicos. La docencia universitaria debe estar vinculada con un proceso de transformación multidimensional que le permita al estudiante contar con un conjunto de atributos personales (conocimientos, habilidades, destrezas, actitudes, valores y disposiciones) para desempeñarse exitosamente en las funciones o tareas de su rol profesional o de trabajo académico.

Las competencias de las personas requeridas para ser exitosas en sus diferentes roles sociales no se sustentan en la simple suma inorgánica de saberes, habilidades y valores; sino en la maestría con que la que se articula, compone, dosifica y pondera constantemente estos recursos y sus resultados ${ }^{1}$.

Se requiere de "mentes bien hechas, que no sólo sepan buscar información, procesarla y transferirla como estrategias de solución para situaciones y problemas con- cretos, sino que también, las puedan transferir de forma flexible y polivalente a nuevos escenarios organizacionales, situaciones y problemas que van surgiendo y que requieren generar conocimientos, aprender y desaprender" (DOMíNGUEZ, 2004). En breve, estas nuevas demandas educacionales requieren revisar los supuestos principales y sus nuevos énfasis.

En particular, la actualización de la docencia debe hacerse cargo de la obsolescencia de formas de enseñanza convencionales sustentadas en supuestos tales como: a) el conocimiento es un cuerpo bien establecido de hechos técnicos que generan una acumulación de resultados que son jerárquicamente organizados y secuenciados para ser divididos y pasados de expertos a novicios, b) el aprendizaje es la adquisición de pedazos aislados de información y habilidades logradas principalmente escuchando, mirando, memorizando y practicando, y c) la enseñanza es la transmisión directa del conocimiento de profesores y de textos a los estudiantes.

Un cambio de énfasis central para la docencia significa pasar de la transmisión de conocimientos de contenidos de tipo académico hacia la construcción por parte de los alumnos y alumnas de nuevas competencias y capacidades que le permitan aprender y seguir aprendiendo en forma permanente. Los contenidos de carácter teórico conceptual no se abandonan sino que se redefinen en función de hacerlos más significativos, actualizados, profundos, generativos de nuevos aprendizajes y pertinentes a las necesidades formativas de los alumnos.

\footnotetext{
' Comisión Nacional para la Modernización de la Educación, Comité Técnico asesor del diálogo nacional sobre la modernización de la educación chilena (1994), que sustentó una Política de Estado en Educación.
} 
El nuevo énfasis está puesto en la enseñanza de procesos, estrategias y habilidades de pensamientos utilizando el conocimiento disciplinario y cultural como medio para el crecimiento personal. El conocimiento se vuelve generativo solo en la medida que no es presentado sin más a los estudiantes, sino que cuando ellos puedan interpretarlos desde sus esquemas cognitivos previos, cuestionar críticamente lo que se les dice y establecer relaciones con otras informaciones. Es decir, un conocimiento genera bases para la construcción de nuevas estructuras de conocimiento cuando puede usarse para resolver problemas, comprender nuevas situaciones y pensar, sentir y actuar competentemente en forma individual y grupal.

La actualización de la actividad docente de modo lograr efectos externos esperados (empleo, ingresos, movilidad social, satisfacción laboral y desarrollo colectivo, entre otros) requieren considerar cambios de las últimas décadas que afectan la labor docente. Por ejemplo, Montero destacó la necesidad de: 1) un cambio de énfasis sobre la preocupación de los procesos internos de las personas para el aprendizaje (antes con el conductismo no eran importantes, en tanto hoy sí lo son); 2) una docencia centrada en la acumulación de conocimientos a una centrada al autoaprendizaje; 3) de la aplicación privilegiada del método expositivo de enseñanza a una variedad de enfoques y situaciones centrados en lograr los aprendizajes de los estudiantes; 4) una enseñanza objetiva y aséptica a una enseñanza disciplinaria que no es valóricamente neutra; 5) el uso de las nuevas tecnologías informáticas y de comunicaciones (TIC'S) que han permitido flexibilizar los accesos a la información, la secuenciación de las actividades de aprendizaje y los procesos de construcción social de los conocimientos; y 6) la evaluación de los aprendizajes referida a la norma (basada en la distribución normal) ha sido cambiada por enfoques tendientes a evaluaciones referidas a criterio (MONTERO, 2004).

Las nuevas expectativas para la docencia están presentes en universidades que poseen un reconocido liderazgo. Por ejemplo, para la docencia de la Universidad de Notre Dame, se destaca una preocupación por: a) un aprendizaje centrado en el estudiante; b) un aprendizaje activo; c) una evaluación auténtica; d) un aprendizaje colaborativo; e) un aprendizaje comunitario; f) diversos estilos de aprendizaje; g) espacios de aprendizajes con tecnología; y, h) aprendizaje a distancia (CLARK, 2002). Otros ejemplos se encuentran en transformaciones más específicas como son algunas presentes en ingeniería, para pasar de una enseñanza centrada en el profesor al estudiante (CALATANO; CATALANA, 2000), y que favorece, también, el desarrollo de competencias transversales centrales para el ingeniero (LACHIVER, 2002).

Investigaciones recientes de la docencia universitaria eficaz destacan la importancia del uso de varios tipos de conocimientos para relacionar la experiencia previa de los estudiantes con el nuevo conocimiento. Los docentes efectivos requieren de conocimientos: disciplinarios, pedagógicos en la disciplina, pedagógicos generales, de sus estudiantes y sobre el aprendizaje, curriculares, de los fines, del contexto y de sí mismo. La aplicación de estos conocimientos se vincula con nuevas funciones y tareas docentes relacionadas con un rol docente no convencional, aspecto que se aborda en la próxima sección. 


\section{Funciones y tareas del nuevo rol docente}

El nuevo rol docente requiere desempeñar exitosamente varias funciones y tareas no convencionales. En esta sección se han privilegiado algunas funciones docentes concordantes con una enseñanza orientada a desarrollar competencias que comprometen variedad de atributos personales y que utiliza ambientes de aprendizajes multimediales para concretar una docencia semipresencial. Finalmente, se relacionan estas funciones con las presentadas en otras experiencias de países desarrollados.

\section{Rol del docente en un aprendizaje semipresencial}

El sistema educativo semipresencial $(\mathrm{SPID} / \mathrm{SP})^{2}$ posibilita una variedad de relaciones entre los estudiantes y los conocimientos, entre estudiantes y entre docentes y estudiantes. Mediante ambientes de trabajo sustentados con tecnología informática y de comunicaciones, tanto profesores como estudiantes tienen una variedad de recursos para el desarrollo de las funciones y tareas claves que comprenden su rol en el sistema.

A modo ilustrativo, los profesores y alumnos cuentan con un diseño y programación de los cursos donde los contenidos y actividades principales de las situaciones de aprendizaje y evaluativas se encuentran accesibles en forma flexible en el espacio y en el tiempo, como también la información general (reglamentos, cronogramas de las pruebas, boletín).

Tanto docentes como estudiantes tienen acceso a las herramientas de comunicación para la interacción individual o grupal en forma sincrónica o asincrónica. Entre los aspectos distintivos, los estudiantes pueden consultar sus dudas en cualquier momento, y los profesores pueden acceder a información relativa al grado de participación de cada estudiante en el proceso y a las calificaciones que ilustren sus estados de avances.

En esta modalidad el docente debe realizar varias funciones que se alejan de su rol convencional. Las caracterizaciones que aquí se presentan se sustentan con la aplicación sostenida de dos programas semipresenciales que están orientando procesos de renovación curricular y metodológica de la USACH. Uno es el Programa de Álgebra para el primer año de Ingeniería ${ }^{3}$, y el otro es el Diplomado en Educación basado en Competencias ${ }^{4}$, aplicados a académicos de varias universidades.

Globalmente, el profesor es un guía, maestro, consejero en el proceso de aprendizaje, a la vez que un analizador crítico, juez, árbitro, reforzador e inspector de los aprendizajes del estudiante. Junto con poseer un amplio dominio de los contenidos, debe reunir varias otras características personales que le permita establecer relaciones interpersonales favorables para el aprendizaje de sus estudiantes tanto en forma presencial como a distancia. La nueva for-

\footnotetext{
2 SPID/SP: Sistema de Perfeccionamiento Interactivo a Distancia, desarrollado y validado por el Centro de Desarrollo, Experimentación y Transferencia de Tecnología Educativa (CEDETEC) de la USACH.

${ }^{3}$ El Programa de Álgebra fue probado experimentalmente durante tres años y, actualmente, lecciones de sus resultados se han utilizado en el MECESUP sobre desarrollo de Ambientes Multimediales de Aprendizajes.

${ }^{4}$ Para obtener mayor información del Diplomado Modular de Educación basada en Competencias, ver sitio disponible en: www.cedetec.cl.
} 
ma de enseñanza reduce la clase expositiva, donde el profesor pasa la materia monopolizando la selección de los contenidos, la secuenciación de las actividades, fijando los ritmos de aprendizaje y las instancias de evaluación de los aprendizajes.

Las clases presenciales en esta modalidad, aprovechan lo que se encuentran en el sistema educativo para el acceso flexible en el espacio y en el tiempo del estudiante, los contenidos, una variedad de actividades y ejercicios para el aprendizaje, múltiples herramientas para acceder a consultas o para la construcción de conocimientos grupales, entre otras. Por ello, en vez de exponer los contenidos en la sala de clase, laboratorio o taller; la labor del docente estará, principalmente, focalizada en actividades no rutinarias para los aprendizajes dirigidas a facilitar la clarificación, profundización, sistematización y proyección de los conocimientos y del desarrollo de competencias por parte de los estudiantes, para un saber hacer con los conocimientos, incluyendo capacidades para lograr su propia autodirección en el aprendizaje y desarrollo profesional.

En las clases no presenciales, los docentes orientan, conducen y retroalimentan a distancia el trabajo individual, grupal o colectivo de sus estudiantes de acuerdo a normas de operación que son conocidas y aceptadas por los estudiantes. En especial, las reglas de operación definen las formas de acceso y normas que regulan la utilización de la variedad de recursos y herramientas que se han puesto al servicio para el aprendizaje de los estudiantes. Estas normas establecen los derechos y obligaciones de estudiantes y docentes en el nuevo escenario.
A continuación, se presentan las funciones y principales tareas que definen el rol del docente en el nuevo sistema:

- Guía: el docente traza con precisión los objetivos que deben lograr los estudiantes, destaca y ejemplifica la importancia de ellos para sus alumnos, y contextualiza la utilización de los recursos que se pueden usar con sus formas de operación para el logro de los aprendizajes. Es el orientador que ilustra las implicaciones del curso y sus componentes en el proceso de crecimiento personal.

- Experto de Contenido: el docente tiene amplio dominio de los contenidos que se abordan en el curso de manera de poder tratarlos con flexibilidad, profundidad y significatividad en la variedad de relaciones de los conocimientos con sus alumnos, sea, en forma presencial o a distancia.

- Facilitador: el docente debe ser el referente académico que resuelve las dudas del estudiante, que le provea de información, explicaciones y orientaciones que contribuyan a superar las barreras para los aprendizajes; es proactivo en asistir a los estudiantes para flexibilizar las secuencias y ritmos de aprendizajes, y para apoyarlos en proyectar los aprendizajes para su desarrollo profesional y personal.

- Moderador: el docente gestiona actividades y dirime en labores grupales, tanto presenciales como virtuales; se distingue por su lenguaje, su trato y carácter empático con sus estudiantes, siendo un modelo para los aprendizajes sociales y efectivos ligados con la autodirección en el aprendizaje disciplinario o multidisciplinario. 
- Controlador de los Procesos: el docente es analizador crítico de las experiencias formativas de los estudiantes, lleva el control de los procesos y utilización de los recursos en forma efectiva y oportuna, de modo de lograr las organizaciones de experiencias de los estudiantes, las cuales han de ser facilitadoras de los aprendizajes esperados.

- Evaluador: el docente lleva el seguimiento de los estudiantes y las evaluaciones de procesos, de resultados y de impactos; junto con las retroalimentaciones, participa activamente en la certificación de los aprendizajes y de las competencias.

\section{El rol docente en una nueva visión sistémica}

Una segunda perspectiva de análisis es aquella que orienta el nuevo rol docente proveniente de la visión esperada de la docencia. Desde el punto de vista sistémico, se ha destacado que la docencia debe hacerse cargo desde que el estudiante ingresa a la universidad, con todas sus fortalezas y debilidades, de la calidad de sus procesos de formación, de sus resultados e impactos para su desarrollo profesional, personal y social, como también, de su fidelización para una educación continua, permanente o a lo largo de toda su vida (MONTERO, 2005).

La visión propuesta fue la siguiente: La Universidad de Santiago de Chile mantiene una oferta educativa dinámica, flexible, con trayectorias múltiples basadas en competencias; pertinente para una educación a lo largo de la vida; fundamentada en continuos estudios de necesidades, antecedentes de las investigaciones y proyectos de desarrollo, estudios de seguimiento de sus egresados; sustentada en ambientes y situaciones para el desarrollo personal y social, con aperturas sistemáticas a nuevas fuentes del saber; con espacios de exploración, descubrimiento e invención; con modelos de experiencias prácticas ligadas a los requerimientos de desempeño para los roles sociales; con certificados de aprendizajes acreditados y valorados socialmente; y basada en los códigos culturales, científicos, tecnológicos, sociales y artísticos propios del contexto y momento histórico.

Desde esta perspectiva, el nuevo rol docente de la USACH se aleja del docente tradicional, cuyo rasgo distintivo fundamental es su conocimiento disciplinario. Al considerar los cuatro subsistemas propuestos orientación y desarrollo profesional, ambientes de aprendizajes, evaluación de aprendizajes, y gestión administrativa - el docente requiere desempeñar varias otras tareas docentes. Entre otras, relacionar las contribuciones formativas de su asignatura con el perfil de egreso y el desarrollo profesional, aplicar repertorios de metodologías facilitadoras de aprendizajes y competencias, de modo que las características de las metodologías interactúen con las características de los estudiantes, aplicar distintas tecnologías propias de los códigos de la modernidad, generar situaciones y ambientes de aprendizajes efectivos, utilizar mecanismos, procedimientos e instrumentos evaluativos que contribuyan al monitoreo y evaluación de aprendizajes y competencias y que posibilite una gestión descentralizada eficaz con unidad.

Para describir con propiedad las nuevas tareas docentes, es fundamental profundizar el análisis. A modo ilustrativo, para 
que el profesor participe con propiedad en los ambientes de aprendizajes debe estar en condiciones de: a) proveer de múltiples perspectivas y representaciones de la realidad; b) proporcionar contenidos y actividades que reflejen las complejidades del mundo real; c) focalizarse en la construcción y no en la reproducción del conocimiento; d) presentar actividades realistas, relevantes y auténticas; e) proveer actividades, oportunidades, herramientas y ambientes que incentiven el autoanálisis, la reflexión, la autoconciencia y la meta cognición; f) promover una práctica reflexiva, g) permitir que el contexto y su contenido dependan de una construcción del conocimiento a través de la negociación social, colaboración y experiencia; h) enfatizar la resolución de problemas, las habilidades de pensamiento de orden superior y la comprensión profunda, e i) alertar de las complejidades del conocimiento, enfatizando las interrelaciones conceptuales y los aprendizajes interdisciplinarios

En suma, varios antecedentes confirman la necesidad de avanzar en una profesionalización de la docencia que compromete varias funciones y tareas propias de un nuevo modelo de docencia. En la próxima sección, a modo de conclusión, se presentan algunos de los principales desafíos que deberían ser abordados sistemática y sistémicamente.

\section{Desafíos y sugerencias}

No es fácil modificar una docencia convencional que generó importantes logros en el contexto de la industrialización, especialmente cuando la media de edad de los académicos es sobre los 50 años. Las grandes resistencias a los cambios se sustentan en un conjunto de cre- encias basadas en evidencias de una dilatada experiencia docente y una cultura institucional con débiles vinculaciones con el medio externo.

El primer y gran desafío es generar una estrategia de cambio realista y efectivo para la docencia, que junto con sensibilizar respecto a la necesidad de los cambios, proporcione herramientas para abordarlos sistémica y sistemáticamente. Las orientaciones e intervenciones deben estar contextualizadas de modo de poder abordar, monitorear y mejorar exitosamente los cambios en la labor docente y su consolidación, mediante un sistema de recompensas institucionales contingentes con la instalación y adopción de las innovaciones.

En esta estrategia de cambio deben considerarse tanto las variables de insumos, procesos, resultados y de impactos de la labor docente. En ella también debe considerarse la coherencia en la toma de decisiones en sus diferentes niveles organizacionales. Por ejemplo, es fundamental contar con una carrera académica y mediciones vinculadas a los aseguramientos de la calidad a nivel institucionales que sean consistentes con las decisiones a nivel carrera, a nivel programas con las presentes a nivel de aula, laboratorio o taller. En breve, la estrategia de cambio de la docencia, junto con responder comprehensivamente a una visión institucional de la docencia, debe responder a los requerimientos específicos de la labor de los profesores con sus estudiantes. Este último requerimiento es un segundo y clave desafío.

En efecto, además de la voluntad política y de la apropiada formulación técnica del proceso de transformación de la docencia, este nuevo desafío consiste en lo- 
grar un alto grado de participación de los diferentes actores. Para ello, junto con sensibilizar a los docentes sobre la necesidad de los cambios, es fundamental realizar acciones que permitan su activa y comprometida participación. En vez de asumir un cambio docente impositivo (por decreto), se ha asumido la importancia de generar un cambio docente mediante la socialización e inducción de asumir los cambios mediante una significativa dosis de convencimiento. El supuesto subyacente que se desea satisfacer es la convicción de que nadie hace bien en lo que no cree.

La Universidad de Santiago de Chile está avanzando en plasmar una estrategia de mejoramiento docente que pretende afectar tanto las decisiones de políticas institucionales como los roles de los docentes en sus aulas, talleres y laboratorios. Entre las varias acciones que dan cuenta de un proceso en marcha, se destacan, a modo ilustrativo, las siguientes: alta prioridad en el análisis y acciones para el mejoramiento de la docencia mediante el Nuevo Plan Estratégico Institucional, discusión y aprobación de un Proyecto Educativo Institucional, y el análisis de una visión sistémica para la docencia del Tercer Milenio.

En un nivel más operativo, actualmente, se realizan varias acciones dirigidas a sensibilizar y empoderar a los académicos para la concreción de las funciones propi- ciadas en el nuevo rol docente. Entre ellas destacan la realización de talleres y el Diplomado en Educación basada en Competencias, el desarrollo de proyectos MECESUP vinculados tanto a los nuevos diseños curriculares como a las innovaciones metodológicas, proyectos de mejoramiento de la docencia -que responden a iniciativas de los académicos o de sus unidades-, la ejecución de experiencias pilotos de desarrollo curricular basado en competencias, y la instalación de diversas conversaciones mediante un sitio we $b^{5}$.

Finalmente, es importante señalar que las diferentes acciones involucradas contribuyen a lograr graduales mejoramientos de la docencia con una creciente participación de los miembros de la comunidad. En un escenario próximo, se espera consolidar la instalación de las nuevas funciones docentes, como también, plasmar las funciones de los estudiantes concordantes con sus nuevos roles y ambientes de trabajo con códigos de la modernidad. En la materialización de las nuevas expectativas para la docencia es fundamental el intercambio de experiencias inter e intra institucionales. Tanto la profesionalización del nuevo rol docente y la concreción del nuevo rol para los estudiantes deberán estar contextualizadas en una cultura de calidad de la docencia que responda oportunamente, con pertinencia, relevancia y calidad a las nuevas y dinámicas demandas educacionales.

${ }^{5}$ Disponible en: <www.innova.cedetec.cl >. Acceso en: 3 agosto 2007. 


\section{Referências}

CALATANO, G.; CATALANA, K. Transformation for teacher-centered to student centered engineering education. West Point, New York: Centre for Enhanced Performance, United States Military Academy, 2000.

CLARK, C. Trends in college teaching style for the 21 st century. Notre Dame, IN: The Kaneb Center, University of Notre Dame, 2002.

DOMÍNGUEZ, G. La sociedad del conocimiento, la formación por competencias y el nuevo rol de la universidad: nuevas necesidades de reestructuración y configuración de los planes de estudio. In: CONGRESO CHILENO DE EDUCACIÓN EN INGENIERÍA, 18., 2004, Santiago de Chile. Anales del... Santiago de Chile: CEDETEC, 2004.

LACHIVER, G. Le développement des compétences en Ingénierie par la pédagogie du problème et du projet: un exemple à I'UdS. Québec, Canada: Doyen de la Faculté de Génie, Université de Sherbrooke, 2002.

MONTERO, P. Roles para la docencia universitaria concordantes con las demandas educacionales del nuevo siglo: reencuentro: análisis de problemas universitarios. Xochimilco: Universidad Autónoma Metropolitana, 2004.

- Una visión para la docencia de la USACH en el contexto del nuevo milenio y algunos desafíos principales. In: JORNADA DE DOCENCIA UNIVERSITARIA, 3., 2005, Santiago de Chile. Disponível em: <www.cedetec.cl>. Acesso em: 3 ago. 2007.

Recebido em: 20/04/2007

Aceito para publicação em: 07/05/2007 